\title{
Atribución de la causalidad y motivación de logro desde una perspectiva evolutiva. Evidencia empírica
}

\section{Jesús Alonso *}

\author{
Universidad Autónoma de Madrid
}

4

L objetivo del presente trabajo es la revisión y análisis de los estudios realizados sobre el desarrollo de la motivación de logro y su relación con el desarrollo de las atribuciones desde los 5 años en adelante, edad en que los niños entran en la escuela. Con ello pretendemos poder determinar cuáles son las implicaciones que las distintas fases del desarrollo por las que pasa el niño en relación con las variables que parecen modular el dinamismo de dicha motivación pueden tener para el desarrollo de la misma.

En relación con nuestro propósito, en primer lugar es preciso tener presente que sólo podremos hablar de motivación de logro en los niños en la medida en que su comportamiento presente las características que permiten definir dicho motivo en los adultos (esperanza y deseo de éxito, miedo al fracaso, establecimiento de un nivel de aspiración y disposición al esfuerzo [Atkinson, 1964], al menos en un grado mínimo. Por otra parte, para que el dinamismo de la motivación de logro llegue a ser el que encontramos en los adultos (Weiner, 1974), es necesaria la intervención de una serie de procesos cognitivos complejos (recogida de información relevante, almacenamiento de información serial en la memoria, síntesis de la misma, capacidad de inferencia, capacidad de deducción, etc.). Cabe esperar, en consecuencia, que en la medida en que el niño no haya adquirido los prerrequisitos cognitivos necesarios, el funcionamiento de la motivación de logro no será el mismo que encontramos en los adultos.

Los psicólogos del desarrollo han dedicado un grado considerable de atención al estudio de los cambios que se producen en la comprensión que el sujeto tiene de su entorno social. En particular, los psicólogos cognitivistas (Kohlberg, 1971) han subrayado el papel que el progreso evolutivo en la capacidad de procesar información y en la capacidad de pensamiento lógico juega en el desarrollo social. De acuerdo con este punto de vista, si las habilidades sociales dependen de la selección e integración de múltiples indicios de información, como ocurre con las habilidades lógicas y matemáticas, el desarrollo social del niño deberá ir en estrecho paralelismo con su desarrollo cognitivo (Feldman y Bush, 1978). Pues bien, creemos que de modo semejante se puede razonar en relación con el desarrollo de la motivación de logro. En este desarrollo se hallan implicadas inferencias que suponen la capacidad de integrar información y la capacidad de razonamiento

* Dirección del autor: Jesús Alonso. Departamento de Diagnóstico Psicológico y Medida. Facultad de Psicología. Universidad Autónoma de Madrid. Cantoblanco. Madrid-34. 
lógico, capacidades cuyo desarrollo es bastante conocido. En consecuencia, es posible utilizar los datos que se poseen sobre el desarrollo cognitivo para predecir el desarrollo del uso de la información en la realización de los juicios implicados en la regulación de la conducta orientada al logro.

Pasamos a continuación, teniendo en cuenta tanto lo que se conoce sobre la estructura y funcionamiento de la motivación de logro en los adultos (Atkinson, 1964; Weiner, 1974, 1980; Fyans, 1980) como el hecho que acabamos de señalar -que se halla condicionado su funcionamiento por el nivel de desarrollo cognitivo en los niños- a revisar los estudios realizados sobre la adquisición de las. mismas.

\section{ESTUDIOS SOBRE LA ADQUISICION Y EVOLUCION DE LAS CONDICIONES QUE DETERMINAN LA EXISTENCIA Y DIN. MISMO DE LA MOTIV. ICION DE LOGRO}

\section{Cuando los resultados se convierten en «éxitos» 0 «fracasos": nacimiento de la motivación de logro}

Heckhausen (1967) recoge una serie de estudios cuyos resultados nos proporcionan, aunque indirectamente, evidencia sobre el desarrollo de las dos primeras condiciones señaladas anteriormente: capacidad para percibir el resultado como éxito o fracaso que implica a' su vez la capacidad de estructurar los distintos niveles de dificultad de la tarea y capacidad de experimentar el resultado como fruto de la propia competencia. Decimos que la evidencia es indirecta porque los estudios no han pretendido directamente determinar si el sujeto es capaz de determinar los distintos niveles de dificultad de la tarea o si considera que el resultado se debe a sí mismo o a otras causas. En la mayoría de ellos se ha observado simplemente el comportamiento de los niños en tareas que permiten el establecimiento de distintos niveles de aspiración, v. gr. construir torres de diversas alturas con cubos, etc. En conjunto, tales estudios parecen indicar que hasta los 3,5 ó 4 años el sujeto no es capaz de considerar el éxito o el fracaso como debido a su propia acción, de modo que pueda experimentar placer por su competencia o vergüenza por su incompetencia. En concreto, Zunich (1964) encontró que los niños de 4 años, comparados con los de 3 , piden ayuda a los adultos con menos frecuencia, no se desaniman ni dejan la tarea tan fácilmente y muestran reacciones más fuertes ante el fracaso. Heckhausen y Wasna (1965) han encontrado resultados semejantes trabajando con imbéciles y teniendo en cuenta su edad mental. Señalan que. siempre que ésta sea superior a los 3,5 ó 4 años, se encuentran todos los signos de la motivación de logro (esperanza de éxito, miedo al fracaso, establecimiento de ún nivel de aspiración y disposición al esfuerzo. Heckhausen y Roelofsen (1962), considerando que el origen de la capacidad de conflicto entre el deseo de éxito y el miedo al fracaso depende de la prominencia relativa de las posibilidades de uno y otro resultado, observaron la conducta de los niños cuando éstas eran iguales. Sus resultados muestran que hasta los 4,5 años los niños confían plenamente en sus posibilidades de éxito. Sólo a partir de esta edad la situación indicada produce conflicto. En otro estudio Hieckhausen y Wagner (1965) encontraron que entre los 3,5 y 4,5 años los niños pueden experimentar conflicto si tienen que elegir entre tareas de grado de dificultad creciente y si tienen que considerar tareas en las que probablemente fallan. Sin embargo, a partir de los 4,5 años son capaces de tomar una decisión que les libre del conflicto. Ya desde esta edad comienzan a mostrar alguna consistencia intraindividual en la preferencia por ciertos niveles de dificultad en el establecimiento de metas. En la misma línea se hallan los resultados obtenidos por McClelland (1958), quien señala que a partir de los 4,5 años se pueden reconocer niveles individuales de motivación de logro en función de la preferencia por determinados niveles de dificultad, cuando la tarea utilizada consiste en tratar de que una estaca clavada en el suelo quede dentro de una anilla que se arroja desde diferentes distancias. McClelland señala que es necesario que los distintos niveles de dificultad puedan verse claramente, pues, al parecer, los niños tienen dificultad en establecerlos a esta edad. 
Heckhausen interpreta el cambio que se produce alrededor de los 4 años y que recogen los estudios anteriores como indicador de que el sujeto adquiere la capacidad cognitiva de estructurar la situación reconociendo diferentes niveles de dificultad de la tarea y reconociéndose como causa de los resultados condiciones mínimas para que podamos hablar de la existencia de motivación de logro. Por nuestra parte, pensamos que hay que hacer algunas precisiones a dicha interpretación. La afirmación de que el sujeto es capaz de reconocerse como causa de los resultados se basa en la observación de las reacciones afectivas ante los mismos y en la renuncia a la ayuda de los adultos, fundamentalmente, observación en base a la cual se infiere que el niño es capaz de realizar una inferencia de tipo causal, aunque sea de modo rudimentario. Aunque los resultados de por sí no son concluyentes ya que cabrían otras esplicaciones, no hay razones que vayan directamente en contra de la interpretación de 'Heckhausen y es posible aceptarla especialmente si tenemos en cuenta que los estudios hechos por Berzonski (1971), Mendelson y Shultz (1976) y Bullock y Gelman (en Gelman, 1978) señalan que en las situaciones que les son familiares los niños de 4 ó 4,5 años son capaces de inferir correctamente la causa de un efecto. Dicho de otro modo: la observación repetida de la covariación entre un hecho y un antecedente del mismo hace posible que el niño considere el antecedente como causa del hecho que le sigue. Parece claro que el niño puede observar tal covariación entre sus acciones y los resultados de las mismas y experimentar, en consecuencia, que él es la causa de los mismos.

2. Cuando lo más difícil vale más: desarrollo de la capacidad para distinguir distintos niveles de incentivo

\subsection{Percepción de distintos niveles de dificultad}

Por lo que se refiere a la afirmación de que el sujeto es capaz de reconocer diferentes niveles de dificultad en la tarea, también hemos de precisar su alcance. El sujeto de 4,5 ó 5 años no es capaz de evaluar el nivel de dificultad de la tarea en base a la información que proporcionaría el conocimiento de los resultados de otros sujetos en la misma. Al menos no se estudia aquí directamente esta posibilidad. La evaluación del nivel de dificultad se hace, al parecer, en base a los indicios proporcionados por la estructura de la tarea. Además, el niño sólo es capaz de establecer estos niveles si los indicios están muy claros (McClelland, 1958). En consecuencia, la posibilidad de experimentar distintos niveles de éxito o fracaso en base al establecimiento de distintos niveles de dificultad es muy reducida. En todo caso, cabe esperar que las implicaciones emo-cionales del éxito o del fracaso sean diferentes a las que encontramos en los adultos en la medida en que los niños no establecen comparaciones con otros al parecer antes de la entrada en la escuela alrededor de los 6 años.

Confirmando de alguna manera lo que acabamos de decir, Veroff (1969) llega a distinguir entre tres tipos de motivación de logro. En primer lugar, distingue lo que denomina «motivación de logro autónoma». Con este concepto hace referencia al hecho de que la dificultad de la tarea y el nivel de aspiración relacionado-con la misma se establecen en base a la comparación de los propios resultados con la estructura de la misma. En este sentido podría decirse que el criterio en base al cual se organiza la motivación de logro es, pues, interno. Veroff especula que los comienzos de este tipo de motivación podrían situarse en torno a los 2 años, dado que el niño que está aprendiendo a andar, a hablar, a subir las escaleras, etc., experimenta continuamente el placer de conseguir algo nuevo. Supone que estas experiencias dan lugar al establecimiento de metas que determinan la existencia de la motivación autónoma de logro. Sin embargo, este tipo de motivación sólo se ha observado a partir de los 4 años mediante la utilización de tareas de nivel de aspiración (Callard, 1964; Heckhausen, 1967). Tras esta edad se incrementa progresivamente hasta alcanzar una primera cota a los 7 años, descendiendo después ligeramente, como muestra el estudio del propio Veroff. En segundo lugar, Veroff señala la existencia de la «motivación de logro social", concepto que hace referencia a la utilización de la información sobre los resultados obtenidos por otros en la tarea para evaluar la propia ejecución y estable- 
cer el nivel de aspiración. Este tipo de motivación aparece cuando el niño ingresa en la escuela, alcanzando una primera cota en torno a los 7-9 años. Por último, estaría la «motivación de logro integrada», concepto que hace referencia a la utilización de los criterios internos o los criterios sociales, según sea apropiado. Esto no se da antes de los 11-12 años.

Por su parte, Ruble, Parsons y Ross (1976) nos informan sobre la adquisición y evolución tanto de la capacidad de experimentar los resultados como éxitos o fracasos como de la capacidad de determinar la dificultad de la tarea en función de la información sobre los resultados que otros sujetos obtienen en la misma. Con tal fin estas autoras realizaron dos experimentos. En el primero de ellos manipularon el tipo de resultado obtenido por los niños (éxito-fracaso), así como la información sobre el número de sujetos que fracasaban o tenian éxito en la tarea con el fin de determinar si tales variaciones influian en la respuesta afectiva de los sujetos y cómo variaba dicha influencia en función de la edad. Para ello utilizaron niños de 6, 8 y 10-11 años. Los resultados que obtuvieron indican, por una parte, que la respuesta afectiva es mayor ante el éxito que ante el fracaso, si bien la magnitud de la reacción tiende a igularse en función de la edad. Al parecer, los niños no parecen sentirse mal ante el fracaso hasta los 6 años. Por otra parte, la reacción afectiva no refleja la influencia del conocimiento de los resultados obtenidos por otros en el grupo de los más pequeños, pero sí en los otros dos grupos. En el segundo de los experimentos se siguió la misma estrategia que en el primero, pero, además, se intentó determinar la influencia de las variables independientes sobre la estimación que los sujetos hacían de su habilidad y del grado de esfuerzo puesto en la tarea. Al igual que en el experimento anterior, los resultados pusieron de manifiesto que la respuesta afectiva es mayor ante el éxito que ante el fracaso, pero esto sólo en el grupo de los pequeños ( 4,0 a 5,10 años). En este experimento no se encontró, sin embargo, que la reacción afectiva reflejase la influencia del conocimiento de los resultados obtenidos por otros niños en el grupo de los mayores (7,5 a 9,5 años). No obstante, utilizaron este conocimiento correctamente para hacer inferencias sobre el grado de esfuerzo realizado. Por otra parte, en relación con la estimación del grado de habilidad y de esfuerzo puesto en la tarea, los resultados indican que, en conjunto, los niños se sienten más hábiles tras el éxito que tras el fracaso, pero sólo a partir de 7,5 años; por lo que se refiere al esfuerzo, ni los pequeños ni los mayores reconocen haber puesto diferente grado de esfuerzo tras conocer el resultado, tanto si es éxito como fracaso. Por último, se encontró una correlación significativa entre habilidad y reacción afectiva ante el resultado del fracaso.

Los resultados anteriores tienen implicaciones importantes a la hora de comprender el desarrollo de la motivación de logro. En primer lugar, antes de los 6-6,5 años la reacción afectiva ante el fracaso es mínima. Independientemente de las causas a que esto pueda deberse, implica que existe una diferencia en relación con la reacción afectiva de los niños mayores de 8 años y de los adultos. Es como si hasta superar esa edad el niño no percibiera la relación que puede haber entre fracaso y autoconcepto. Tal diferencia implica, en consecuencia, que el miedo al fracaso será diferente en unos y otros sujetos y su influencia sobre la motivación de logro será distinta. En segundo lugar, l̃os sujetos más pequeños se consideran tan hábiles cuando tienen éxito como cuando fracasan. Esto parece implicar que las connotaciones del concepto de habilidad no son las mismas para los niños que son menores de 6-6,5 años que para los que tienen más de dicha edad. En consecuencia, el papel que la atribución de un resultado a la habilidad puede desempeñar como mediadora en la dinámica de la motivación de logro, si es que desempeña algún papel, será distinto antes y después de la edad mencionada. Es necesario, no obstante, determinar las connotaciones que el concepto de habilidad tiene para los niños antes de los 6,5 años. A este respecto los resultados nos ofrecen una pista: la evaluación del grado de esfuerzo y de habilidad puestos en la tarea van paralelos en el grupo de los más pequeños. Esto nos lleva a preguntarnos en qué medida los niños distinguen entre ambos conceptos. Mientras no distingan entre ellos no podremos esperar, como ya hemos dicho, que su papel en la dinámica de la motivación de logro sea el mismo que desempeñan en los adultos. En tercer lugar, la diferencia 
que hay entre los sujetos más pequeños y los mayores de 8 años en cuanto al uso de la información relativa a los resultados de otros sujetos en la tarea cara a determinar su grado de dificultad implica que es probable que haya diferencias en la reacción afectiva antes y después de dicha edad. Queda, sin embargo, por determinar el modo preciso en que estas diferencias - de algún modo identificadas en el primer experimento - se ven influidas por el uso de la información relativa a los otros sújetos.

Paralelamente al estudio que acabamos de comentar, Shaklee (1976) ha estudiado el desarrollo de la capacidad de percibir distintos niveles de dificultad en la tarea en función de la información relativa a los resultados obtenidos por otros sujetos en la misma tarea y el de la capacidad de inferir el grado de habilidad del sujeto a partir de la secuencia de resultados obtenidos en la tarea. La adquisición de tales capacidades supone como minimo, según Shaklee, la presencia de otras dos: a) la capacidad de integrar información, y b) la capacidad de relacionar el tipo de información que se ha integrado bien con la noción de facilidad-dificultad de la tarea, bien con la noción de habilidad, según los casos. Por otra parte, Inhelder y Piaget (1964) han señalado que la integración de varios elementos de información es una capacidad que corresponde al período de las operaciones concretas. Los niños de niven preoperatorio tienden a centrarse en un solo indicio, y esto les impide el poder integrar varios a la hora de hacer juicios. En consecuencia, Shaklee predijo que se produciría una diferenciación creciente en función de la edad en la capacidad de evaluar tanto la dificultad de la tarea como el nivel de habilidad como fruto del incremento en la capacidad de integrar información. Con el fin de comprobar su predicción realizó dos experimentos. En el primero utilizó sujetos de 4 a 5 , de 5 a 6,5 y de 7,5 a 8,5 años de edad. Por lo que se refiere a la capacidad de estimar la dificultad de la tarea, los resultados pusieron de manifiesto que la información relativa a los resultados de otros sujetos influye en la estimación del grado de dificultad a todas las edades, pero se produce además una mayor diferenciación de los juicios con la edad. Resultados análogos encontró en relación con la capacidad de estimar la habilidad. Estos resultados, sin embargo, no implican que la menor precisión de los pequeños al realizar sus juicios se deba a la incapacidad de sintetizar la información. Podría ocurrir que fuesen capaces de integrar la información adecuadamente, pero que no utilizasen el resultado de esta operación para inferir la dificultad de la tarea o la habilidad del sujeto. Para comprobar la validez de ambas posibilidades realizó un segundo experimento análogo al anterior, utilizando sujetos de 4 a 5 y de 5 a 6,5 años. En este experimento se daba al sujeto una síntesis de la información presentada antes de que diera su respuesta. En consecuencia, si los juicios mejoraban se podría concluir que la limitación se hallaba en la capacidad de integrar información. El caso contrario supondría que ambos requisitos son necesarios para realizar un juicio correcto y ambos se hallaban sin desarrollar a los 6,5 años. Los resultados, sin embargo, no permiten una conclusión clara, ya que en el caso de las inferencias sobre la habilidad se produjo una mejora, pero no en el caso de las inferencias sobre la dificultad de la tarea.

Nicholls (1978 b), en un experimento semejante al de Shaklee con sujetos de preescolar, $10^{\circ}, 2 .^{\circ}$ y $3 .^{\circ}$, encontró que ya a partir de los 6,5 años el 62,5 por 100 de los sujetos es capaz de percibir diferentes niveles de dificultad, porcentaje que se eleva al 75 por 100 al año siguiente. Sus resultados matizan, pues, los obtenidos por Shaklee.

A nuestro juicio los resultados de los dos estudios anteriores tienen las siguientes implicaciones en relación con la motivación de logro:

a) Dado que hasta los 6,5-7 años los niños no parecen capaces de estructurar espontáneamente los distintos niveles de dificultad de la tarea a partir de la información sobre los resultados obtenidos por otros sujetos, tampoco serán capaces de diferenciar los distintos niveles de incentivo que tal estructuración posibilita descubrir. En consecuencia, la respuesta afectiva ante el éxito o el fracaso hasta esa edad será menos diferenciada que a edades posteriores, como veíamos al comentar el estudio de Ruble, Parsons y Ross (1976).

b) Dado que la capacidad de inferir el grado de habilidad se halla limitada hasta los 6,5-7 años por la incapacidad de integrar información, la atribución del éxito o 
el fracaso a esta causa no tendrá las mismas implicaciones que tras dicha edad. Antes de los 7 años es probable que la habilidad no se considere como algo estable e incluso, como veíamos en el trabajo de Ruble, Parsons y Ross, que no se vea como algo independiente del esfuerzo.

c) Si hasta los 6,5-7 años el niño se halla limitado en su capacidad de integrar información serial, probablemente no será capaz de establecer expectativas realistas, ya que éstas deben tener en cuenta la historia de éxitos y fracasos en la tarea. De hecho, como parecen indicar los resultados señalados por Heckhausen (1967) y Parsons y Ruble (1972), ni siquiera el hecho del éxito o el fracaso parecen afectar a las expectativas de éxito en los intentos que siguen inmediatamente a los mismos.

d) Como consecuencia de lo que acabamos de señalar en el punto anterior, cabe esperar que si preguntamos a sujetos menores de 7 años que a qué atribuyen resultados que concuerdan con, o que son contrarios a la historia de resultados anteriores, las respuestas no concuerdan con las predicciones que implica el modelo de Weiner (1974), esto es, resultados consistentes con la historia anterior probablemente no serán atribuidos sistemáticamente a causas estables ni los resultados inconsistentes a causas variables. Esta suposición no implica que pensemos que los niños no explican los resultados que obtienen atribuyéndolos a distintas causas. Tan sólo suponemos que las atribuciones - caso de darse- serán distintas en los sujetos mayores y menores de 7 años.

\subsection{Percepción de distintos niveles de incentivo}

La tercera condición necesaria para el desarrollo de la motivación de logro era que el sujeto sea capaz de percibir la relación que hay entre nivel de dificultad y grado de incentivo. Ya hemos anticipado, a partir de los resultados comentados hasta aqui, que es probable que tal asociación no se dé antes de los 6,5-7 años. Nicholls (1978 b) ha investigado di:rectamente el desarrollo de dicha capacidad. Los resultados encontrados han puesto de manifiesto que aunque algunos sujetos $(6$ por 100$)$ son capaces de perci- bir que las tareas difíciles tienen más valor ya a los 5 años, el número de sujetos que posee tal capacidad no alcanza un porcentaje notable hasta los 7 u 8 años (56 por 100 y 68 por 100 , respectivamente). Parece, pues, que hay un ligero desfase entre la adquisición de la capacidad de percibir el nivel de dificultad de la tarea y la comprensión de que las tareas más difíciles tienen mayor valor incentivo. Es probable que el desarrollo de dicha asociación dependa de que el sujeto sea capaz de inferir que el éxito o el fracaso, según sea de difícil la tarea en que se obtienen, son indicativos de distinto grado de habilidad. Esto es, es probable que la asociación "nivel de dificultad-incentivo" dependa de la asociación «nivel de dificultad-resultadonivel de habilidad».

Los resultados anteriores implican que en los niños menores de 7,5-8 años probablemente se dará una preferencia por las tareas fáciles independientemente de que sus expectativas en las difíciles sean altas. También es probable que persistan menos ante problemas insolubles. Esto es, la variación objetiva de la dificultad de la tarea no alterará la intensidad de la conducta orientada al logro. De hecho, los resultados de un trabajo realizado por nosotros no publicado (Alonso Tapia, 1983 a) apuntan en esta dirección. Sometimos a 180 niños de $1 .^{\circ}, 3 .^{\circ}$ y $5 .^{\circ}$ de EGB, 60 por nivel, a una prueba de persistencia en la resolución de problemas insolubles. Se daba a cada sujeto un minuto por problema, al terminar el cual se le indicaba si el problema estaba bien o mal resuelto. Para el análisis de los resultados, y dado que las distribuciones no eran normales, utilizamos la prueba U de Mann-Whitney. Los resultados encontrados son los siguientes:

$$
\begin{aligned}
& 10^{\circ}-3 .^{\circ}: z=-4,06^{\circ} \\
& 10^{\circ}-5 .^{\circ} z=-5,26^{\circ} \\
& 30^{\circ}-5 .^{\circ}: z=0,85 \text { N.S. } \\
& 0 \text { = resultado significativo al } 5 \text { por } 100
\end{aligned}
$$

Parece, pues, que en relación con la persistencia en la tarea a pesar del fracaso existen diferencias significativas entre los sujetos de $1 .^{\circ}$ (6-6,5 años) y los de los restantes grupos. Estos resultados son los que cabía esperar a partir de las implicaciones de los estudios anteriormente comentados y parecen confirmar la falta de asociación en los más pequeños entre nivel de dificultad $y$ nivel de incentivo. 
3. El esfuerzo es elogiable, pero ser listo es valioso: adquisición de los conceptos de habilidad y esfuerzo y comprensión de sus relaciones mutuas

Por lo que se refiere a la necesidad de que el sujeto comprenda la relación compensatoria que hay entre habilidad y esfuerzo, Karabenick y Heller (1976) han realizado un estudio directamente relevante, aunque veremos también otros que aportan evidencia indirectamente. Seguin Heider (1958), el esfuerzo que hay que poner en cada tarea varía directamente con el grado de dificultad de la misma e inversamente con el grado de habilidad del sujeto si el resultado se mantiene constante. Los autores mencionados analizaron el grado en que niños de distintas edades llegan a comprender dicha relación. El estudio es especialmente importante, ya que dicha comprensión influye en la realización de atribuciones $y$, consiguientemente, en los efectos de las mismas sobre la motivación de logro, según señala Weiner (1980). Se urilizaron sujetos de 7,9 y 11 años de edad. Los procedimientos utilizados pueden resumirse básicamente en contar a los niños una historia en la que se describen dos sujetos que varían en nivel de habilidad, preguntando a continuación a los niños cuál de los dos sujetos tendrá que esforzarse más para resolver un rompecabezas, y en contarles otra en que los dos sujetos se han esforzado de modo diferente para resolver la tarea, preguntando a continuación cuál de ellos es el más hábil. Los resultados pusieron de manifiesto que ya desde $1 .^{\circ}$, el 63 por 100 de los sujetos es capaz de inferir correctamente la habilidad a partir de los indicios de esfuerzo y el 91 por 100 el esfuerzo a partir de los indicios de habilidad, siendo los porcentajes encontrados prácticamente iguales en los tres grupos de edad.

A nuestro juicio, estos resultados completan los datos que sobre la adquisición del concepto de habilidad nos proporcionan los estudios anteriores, aunque es de lamentar que no se hayan incluido en este estudio sujetos menores de 6,5 años. Los estudios anteriores señalaban que a partir de esta edad los niños son capaces de inferir el grado de habilidad a partir del patrón de resultados del sujeto; por otra parte, el estudio realizado por Karabenick y Heller muestra que a partir de los 7 años son además capaces de ver que existe una relación compensatoria entre habilidad y esfuerzo. No obstante, son insuficientes aún para conocer la eqlución de los conceptos de habilidad y esfuerzo, ya que estos conceptos tienen, además, otras connotaciones.

Por otra parte, los resultados anteriores muestran la existencia de un desfase en la comprensión de las relaciones entre habilidad y esfuerzo, dado que parece más fácil inferir el esfuerzo a partir de la información sobre la habilidad que a la inversa. No está claro el porqué de este desfase. En cualquier caso este resultado implica que para un elevado porcentaje de sujetos (40 por 100 ) la realización de inferencias relativas al grado de habilidad, probablemente no tendrá lugar conforme al modelo adulto puesto de manifiesto por Weiner (1980) y sugerido por Heider (1958). Situaciones análogas, en consecuencia, no tendrán las mismas implicaciones motivacionales antes y después de los 11-12 años.

Nicholls (1978 a) ha estudiado en niños de 7,10 y 13 años la evolución de las atribuciones al esfuerzo, la habilidad y la suerte, haciendo variar exclusivamente la información relativa al grado de esfuerzo puesto en la tarea por los sujetos observados y la relativa al resultado. Debido al procedimiento utilizado, las atribuciones a la habilidad y a la suerte implicaban la comprensión de la relación compensatoria entre esfuerzo y habilidad y la comprensión de que el grado en que el resultado de una tarea puede deberse a la habilidad o a la suerte depende de la naturaleza de la tarea, o, dicho de otro modo, de la naturaleza básicamente aleatoria o no de los resultados de la misma. En este caso, la tarea en que trabajaban los sujetos observados consistía en la resolución de problemas de matemáticas. Los resultados pusieron de manifesto que las atribuciones al esfuerzo covarían con el grado de esfuerzo observado, y que esta covariación se hace más marcada con la edad. Por otra parte, resultados inconsistentes con la falta de esfuerzo sólo se atribuyen a la habilidad en los sujetos mayores. En los demás sujetos estos resultados se atribuyen en general a la suerte. En cualquier caso, las atribuciones 
al grado de habilidad tienden a decrecer con la edad y a incrementarse las atribuciones al grado de esfuerzo o de suerte.

A nuestro juicio, los resultados anteriores ponen de manifiesto que, a diferencia de lo que señalaban Karabenick y Heller, la capacidad de inferir el grado de habilidad de un sujeto a partir de los indicadores de su grado de esfuerzo y del resultado obtenido no se pone de manifiesto hasta pasados los 10 años de edad cuando se ofrecen al sujeto más alternativas para explicar los resultados. Da la impresión de que ante datos inconsistentes, los sujetos reconocen que no se pueden atribuir al grado de esfuerzo y, en ausencia de una comprensión clara de la relación compensatoria entre habilidad $y$ esfuerzo, así como de la ausencia de comprensión clara de la relación entre suerte-habilidad y naturaleza aleatoria-no aleatoria de los resultados de la tarea, echan mano cuando se les pide una explicación de lo primero de que disponen.

Lo que acabamos de decir comentando los resultados de Nicholls (1978 a) sugiere que el tipo de procedimiento utilizado para estudiar la realización de atribuciones puede producir resultados significativos y que, sin embargo, no reflejen la realidad. Pensamos que es necesario utilizar procedimientos en los que la información proporcionada sea múltiple y lo más semejante posible a la que el sujeto tiene que procesar en la situación real, y que las alternativas ofrecidas para explicar los resultados han de ser también múltiples. Esto si se quiere estudiar la capacidad del sujeto de realizar inferencias causales de modo semejante a los adultos. Sin embargo, creemos que será necesario en una segunda fase de investigaciones y con el fin de determinar el comportamiento real de los sujetos no sugerirles las posibles causas $e$ incluso no sugerirles ni siquiera la explicación de los resultados, sino sólo que manifiesten en voz alta sus pensamientos durante y después de la tarea, con el fin de determinar cuándo y cómo los niños buscan la explicación de los resultados. En este sentido se orientan los trabajos de Wong y Weiner (1981) y Diener y Dweck (1978).

Aparte de las implicaciones metodológicas señaladas, los resultados del estudio descrito tienen implicaciones " teóricas seme jantes a las del estudio de Karabenick y Heller, esto es, la realización de inferencias relativas al grado en que el nivel de habilidad del sujeto es responsable de unos resultados será diferente antes y después de los 12 años e influirá de modo distinto en la motivación de logro.

En un estudio complementario del anterior, Nicholls (1978 b) completó su examen de la evolución de las atribuciones al esfuerzo y a la habilidad, utilizando esta vez sujetos de 5-6, 7-8 y 9-10 años de edad. La metodología fue análoga a la del estudio anterior, esto es, se utilizaron como variables independientes la información relativa al grado de esfuerzo puesto en la tarea y el resultado obtenido. Los resultados que obtuvo pusieron de manifiesto que el razonamiento del niño sobre la habilidad y el esfuerzo pasa por cuatro niveles sucesivos:

a) Nivel 1 (5-6 años).-Los niños no distinguen esfuerzo y resultado como causa y efecto. Dan explicaciones tautológicas y no distinguen habilidad, esfuerzo y resultado como dimensiones separadas. Se suelen centrar en el resultado (consideran que los que obtienen puntuación más alta se han esforzado más -aunque no sea así- y son más listos) o en el esfuerzo (los que se esfuerzan más son más inteligentes, aunque su puntuación sea menor).

b) Nivel 2 (7-8 años).-Los niños distinguen esfuerzo y resultado como causa y efecto. Se considera el esfuerzo como causa primaria de los resultados. Pero no sugieren la habilidad, entendida como capacidad que puede incrementar o limitar la efectividad del esfuerzo, como causa de los resultados.

c) Nivel 3 (9-10 años).-Se reconoce que el esfuerzo no es la única causa de los resultados. Pero el concepto de habilidad sólo se usa de manera intermitente.

d) Nivel 4 (más de 10 años).-Se usa sistemáticamente el concepto de habilidad de modo correcto y se infiere también de modo correcto a partir de los indicadores de esfuerzo y del resultado.

A nuestro juicio, la validez de los resultados de este estudio se halla condicionada por la metodología utilizada. Dado que los niños para hacer sus inferencias sobre habilidad debían apoyarse en los indicadores de esfuerzo, los resultados pue- 
den aceptarse como indicadores válidos de la relación entre esfuerzo y habilidad. Sin embargo, no nos informan de cómo evoluciona la capacidad del niño de inferir habilidad a partir de la información que normalmente sirve de base para esta inferencia y que viene dada por la historia de resultados del sujeto. Shaklee (1976) encontró que los niños adquieren esta capacidad alrededor de los 7 ó 7,5 años, aunque si la historia de resultados es muy homogénea pueden distinguir diferencias en habilidad a partir de los 4,5 ó 5 años. En consecuencia, si queremos alcanzar un conocimiento más detallado de la capacidad de los niños para usar las distintas atribuciones será necesario tener en cuenta que éstas dependen al menos en parte del tipo de indicios de información que proporcionamos a los sujetos. Por tanto, habrá que multiplicar dichos indicios del modo que ya hemos indicado al comentar el estudio anterior de Nicholls (1978 a).

La penúltima condición necesaria para que la dinámica de la motivación de logro fuera semejante a la encontrada en los adultos se refería a la necesidad de que el sujeto posea esquemas causales semejantes a los de los adultos. Anteriormente, al describir el modelo de Weiner (1974, 1980) sobre la relación entre atribución de la causalidad y motivación de logro ', hemos descrito los esquemas causales como «creencias relativamente estables sobre el modo en que se combinan ciertas causas para producir determinados efectos». También describimos entonces a modo de ejemplo dos de estos esquemas, el esquema múltiple suficiente y el esquema múltiple necesario. El uso de éstos y otros esquemas parece que sólo es posible en la medida en que los niños manejan ciertas reglas de inferencia. De ahí que el estudio evolutivo del uso que los niños hacen de tales esquemas vaya ligado en los trabajos que recogemos a continuación al estudio de la evolución de la capacidad de manejar determinadas reglas de inferencia.

El principio más básico que parece hallarse a la base de cualquier inferencia causal y del uso de cualquier esquema es el "principio de covariación»: para que una realidad (persona, hecho, suceso, objeto, etc.) pueda ser considerada como causa de un efecto es necesario que la aparición de éste vaya normalmente precedida de la presencia de dicha realidad. Los estudios anteriormente citados de Berzonski (1971), Mendelson y Shultz (1976) y Bullock y Gelman (en Gelman, 1978), y el estudio de Shultz y Mendelson (1975) han puesto de manifiesto que ya desde los 3 años los niños son capaces de utilizar la covariación existente entre ciertos datos para hacer inferencias sobre sus causas.

Kun, Parsons y Ruble (1974) han estudiado evolutivamente el uso que los niños de 5 a 10 años hacen del esquema "gradual». Este término hace referencia a la situación en la que el que realiza una atribución cree que la variación en la fuerza de una causa produce una variación en la intensidad del efecto. Para determinar el grado en que los niños utilizaban este esquema se les proporcionó información sobre el grado de habilidad o de esfuerzo de unos sujetos y se les pedía que hiciesen predicciones sobre la ejecución posterior de tales sujetos. Los resultados pusieron de manifiesto dos cosas. En primer lugar, que los niños de todas las edades observadas utilizaban este esquema, considerando que la ejecución es función monotónica creciente de las dos causas facilitativas de la misma, habilidad y esfuerzo. En segundo lugar, que aunque los niños de 5 años pueden utilizar el principio en relación con cada uno de los indicios por separado, no tienen en cuenta el modo en que interactúan modulando su significado.

Como complemento del estudio anterior, Kun (1977), utilizando su jetos de 6,8 y 10 años, ha examinado el uso que los niños hacen del esquema gradual no para predecir, sino para explicar unos resultados dados. El procedimiento utilizado consistió en contar a los niños una serie de historias en las que se les proporcionaba información sobre el grado de esfuerzo o habilidad del sujeto que presentaban, intentando resolver un rompecabezas y sobre el resultado que obtenía. A continuación se pedía a los niños que juzgase el grado en que la causa sobre la que no se proporcionaba información en la historia se hallaba presente en el sujeto. Kun partió del supuesto de que la utilización correcta del «esquema gradual» implica el manejo de dos reglas de inferencia:

\footnotetext{
1 Véase el artículo introductorio de este dossier.
} 
a) El principio de «covariación de la magnitud", según el cual «un cambio en el grado de un efecto se relaciona positivamente con un cambio en el grado de una causa facilitativa".

b) El principio de "compensación", según el cual, si un efecto permanece invariante, un cambio en la magnitud de una causa va acompañado por otro cambio compensador en la magnitud de una segunda causa.

Los resultados encontrados por Kun muestran que desde los 5,5 ó 6 años los niños manejan el primero de los dos principios señalados. Por otra parte, indican que el uso del esquema de compensación cambia con la edad. En concreto, reflejan la asistencia de una asimetría a la hora de hacer las distintas inferencias: no es lo mismo inferir esfuerzo a partir de la información relativa a la habilidad del sujeto que a la inversa; lo segundo parece más difícil y no aparece ni en los sujetos de más edad, mientras que los niños se muestran capaces de inferir correctamente el esfuerzo a partir de la habilidad desde los 8 años aproximadamente. Antes de esta edad, en el grupo de 6 años, se encontró que los niños utilizan consistentemente un esquema distinto al de los mayores a la hora de inferir el esfuerzo a partir de la información sobre la habilidad: consideran que a mayor esfuerzo corresponde mayor habilidad, fenómeno que Kun considera debido a la utilización de un esquema "halo».

En un segundo experimento publicado conjuntamente con el anterior, Kun trató de esclarecer el desarrollo del principio de compensación. Para ello distinguió entre:

a) Un «principio de compensación directa", según el cual, si un resultado no varía, el cambio en la fuerza de una causa inhibitoria (v. gr., dificultad de la tarea) se relaciona positivamente con el cambio en la fuerza de una causa facilitadora.

b) Un «principio de compensación inversa", según el cual, si un resultado no varía, el cambio en la fuerza de una causa facilitadora se relaciona negativamente con el cambio en la fuerza de otra causa facilitadora.

Tras realizar esta distinción puso a prueba la hipótesis de que los sujetos manejarian el primer principio antes que el segundo, dado que el primero supone tener en contra la covariación directa entre dos causas y el segundo la covariación inversa. Adicionalmente supusieron, en base a resultados anteriores (Kun y Weiner, 1973; Parsons y Ruble, 1972), que ambos esquemas de compensación se desarrollarían antes en relación con el éxito que con el fracaso. Ambas hipótesis se vieron confirmadas por los resultados. El manejo del principio de compensación directa es usado ya por los sujetos de 5-6 años, pero su utilización mejora notablemente a partir de los 8-9, mientras que el dominio del esquema de compensación inversa no se logra hasta casi los 12 años.

Los estudios de Berzonski, Shultz y Mendelson, Mendelson y Shultz, Bullock y Gelman, Kun, Parsons y Ruble, y Kun, que acabamos de comentar, y los anteriormente comentados de Nicholls y Karabenick y Heller han puesto de manifiesto diferentes aspectos de la adquisición de los esquemas causales, en concreto el esquema gradual. Los niños parece que son capaces de usar el principio de covariación desde los 3 años; el principio de covariación de la magnitud desde los cinco; el principio de compensación directa desde los 6 , aunque de modo notabie sólo a partir de lós 8-9; y el pinincipio de compensación inversa a partir de los 11-12. Esto se ha puesto de manifiesto en las inferencias sobre la participación del esfuerzo a partir del grado manifiesto de habilidad y a la inversa. Parece, pues, que hasta los 11-12 años los niños no llegan a ponderar el peso de una causa en relación con el peso de otras causas posibles, como hacen los adultos. Cabe esperar, por consiguiente, que sólo a partir de esta edad la influencia de las atribuciones en la motivación de logro sea equivalente en unos y en otros.

Los estudios anteriores han sacado a la luz dos hechos cuya explicación es importante si queremos comprender cuál puede ser la influencia de las atribuciones sobre la motivación de logro antes de la edad señalada. El primero de ellos es el empleo de lo que Kun (1977) ha denominado "esquema halo" - la creencia de que a mayor esfuerzo corresponde mayor habilidad y a la inversa- en los niños de 5 y 6 años. Se han sugerido varias hipótesis para explicar este hecho, pero hasta ahora ninguna de ellas ha sido puesta a prueba. En primer lugar, se ha sugerido que la prác- 
tica en una tarea que conlleva más esfuerzo suele producir una mejora en la habilidad, y que la experiencia de este hecho podría ser la base del efecto halo cuando hay que inferir habilidad a partir de los indicadores de esfuerzo. En segundo lugar, y desde la perspectiva de las teorías de la consistencia cognitiva, se ha señalado que los individuos cuya estructura cognitiva es más simple -y éste sería el caso de los niños de 5 y 6 años- prefieren införmación consistente y tienden a formar impresiones afectivamente univalentes de los demás, lo que daría lugar en el caso que nos ocupa al esquema halo. Caso de ser cierta la primera hipótesis, la habilidad aparecería como una causa variable y bajo control del sujeto, con lo que las implicaciones de esta atribución sobre la ejecución futura serían justamente las contrarias a las que tiene en los adultos. En el caso de la segunda hipótesis, no podemos saber si las implicaciones son iguales o diferentes.

El segundo hecho puesto de manifiesto por los estudios anteriores es la existencia de una asimetría entre las inferencias sobre habilidad a partir de los indicios de esfuerzo y las inferencias sobre esfuerzo a partir de la información sobre el grado de habilidad. A pesar de que ambos problemas son simétricos desde un punto de vista lógico, las inferencias sobre el grado de esfuerzo tienden a ser más conformes con el empleo del esquema de compensación inversa y menos al empleo del esquema halo que las inferencias sobre el grado de habilidad, y ello a todas las edades estudiadas. Este hecho indica que los niños, desde los 5 años, conciben la habilidad y el esfuerzo como entidades psicológicas de algún modo diferentes. No obstante, no se ve claro en qué puede consistir la diferencia entre el esfuerzo y la habilidad que da lugar a este hecho. Se ha sugerido la hipótesis de que la asimetría señalada puede que refleje experiencias diferentes de cómo una causa cambia en función de la otra. En concreto, puede que se deba a la experiencia de que el esfuerzo está bajo control voluntario y la habilidad no. De ahí que los niños perciban qué cambios en el grado de esfuerzo puedan compensar cambios en el grado de habilidad, mientras que no es tan fácil que perciban lo contrario. Si esta hipótesis es cierta, implica que los niños estudiados y los adultos coinciden en ver la habilidad como «no controlable». En la medida que esto sea así, las implicaciones de esta atribución serán semejantes en niños y adultos.

\section{4. «Creo que voy a conseguirlo». ¿Cuándo puede basarse esta afirmación en expectativas realistas?}

La última de las condiciones necesarias para que se dé el funcionamiento de la motivación de logro que encontramos en los adultos es que el sujeto sea capaz de relacionar el patrón de resultados obtenidos y sus causas con sus posibilidades de éxito futuro, o lo que es igual, es necesario que sea capaz de formar expectativas realistas. Ya dijimos que esta capacidad depende en parte de que el sujeto sea capaz de cuantificar de algún modo la información relativa a su historia de resultados y de resumirla o sintetizarla estableciendo el balance de éxitos y fracasos. En relación con este punto ya hemos visto, al comentar los trabajos de Shaklee (1976) y Nicholls (1978 b) que hasta los 6,5 ó 7 años, la capacidad de integrar diferentes indicios de información se halla muy limitada y que, en consecuencia, no parece probable que el sujeto sea capaz de formar expectativas realistas, basadas en su historia de éxitos y fracasos. Por otra parte, los resultados encontrados por Heckhausen (1967) y Parsons y Ruble (1972) indican además que ni siquiera el hecho del éxito o el fracaso parecen influir en las expectativas.

Nicholls (1975) ha estudiado la formación de expectativas en sujetos de edades comprendidas entre 9,5 y 10,5 años. Los resultados de su estudio muestran que a los 9 años, tanto los niños como las niñas son capaces de integrar la información relativa a los resultados obtenidos en sucesivos ensayos de práctica en la tarea y de utilizar la sintesis resultante para formar expectativas de acuerdo con la misma. Adicionalmente encontró que los chicos suelen tener mayores expectativas que las chicas cuando a una serie de éxitos le sigue un fracaso, pero no en otros casos, lo que podria deberse al tipo de atribuciones que median en uno y otro caso.

Posteriormente Parsons y Ruble (1977), basándose en estudios anteriores (Wei- 
ner, 1974; Heckhausen, 1967; Parsons y Ruble, 1972; Parsons, Ruble, Hodges y Small, 1976) estudiaron la formación de expectativas en sujetos de 3,5 a 5 años, de 6,5 a 8 años y de 9,5 a 11 años, poniendo a prueba las siguientes hipótesis: a) las expectativas varían de forma monotónica en relación con el número de éxitos o fracasos precedentes obtenidos en la tarea en los dos grupos de chicos mayores, pero no en el de los más pequeños; $b$ ) las expectativas disminuyen con la edad, dada la mayor experiencia del sujeto; c) las niñas manifiestan expectativas menores que los niños en iguales condiciones, excepto en el grupo de los más pequeños. Los resultados confirmaron plenamente las hipótesis. En relación con la primera de ellas, que es la que más nos interesa, pusieron de manifiesto, en primer lugar, que las expectativas varían en función de la edad y del tipo y número de resultados anteriormente obtenidos. Se encontró que los niños más pequeños (3,5-5 años) a la hora de formar expectativas no usan la información procedente de los sucesivos resultados anteriores previamente integrada de algún modo, lo que es congruente con los resultados de Heckhausen (1967), Parsons y Ruble (1972), Shaklee (1976), y con los que posteriormente ha encontrado Nicholls (1978 b). En el grupo de 6,5 a 8 años las expectativas variaron monotónicamente con la historia de resultados, mientras que el grupo de los mayores sólo lo hicieron así en relación con los éxitos, pero no con los fracasos.

A nuestro juicio, las implicaciones que los resultados anteriores parecen tener para el desarrollo de la motivación de logro son las siguientes. Si los sujetos menores de 6 años no son capaces de integrar datos de información que se han producido de forma secuencial, esto es, separados en el tiempo, no parece que pueda hacerse una idea exacta sobre sus capacidades, sobre su habilidad, si consideramos que ello implicaría ser capaz de percibir la constancia en el éxito o el fracaso en una tarea en la que el resultado depende del propio sujeto, percepción que requeriría la integración de información a que aludíamos. Esto no significa que el sujeto no use el término habilidad cuando se le pide que indique las posibles causas de los éxitos o los fracasos, como veremos (Frieze y Snyder, 1980), sino que tal término no tiene las mismas implicaciones que en el caso de los adultos. El hecho señalado podría explicar que el sujeto sea incapaz de ver las implicaciones que la secuencia de resultados obtenidos en el pasado tiene para la acción que sigue: al no depender los resultados de una causa estable (percibida como tal), el resultado de cada intento no tiene por qué relacionarse con los anteriores. Esto, sin embargo, plantea una serie de interrogantes aún no resueltos. En primer lugar, ¿qué es lo que determina las expectativas de los sujetos a estas edades? ¿basta que perciba que lograr algo es mejor que no-lograrlo para que se crea capaz de hacerlo? ¿o tal vez dependen sus expectativas de las expectativas que los adultos tienen sobre él y que le transmiten mediante expresiones como «iqué tonto eres!» «iqué listo eres!», etc., y que el sujeto acepta de manera acrítica? Se necesita investigación adicional para responder a estas cuestiones.

\section{Cuando la suerte es la causa del éxito: implicaciones para la motivación de logro del desarrollo de la noción de azar}

Ninguno de İos estudios recogidos hasta el momento se ha ocupado de analizar la evolución de la comprensión del concepto de suerte o azar. Sin embargo, sabemos (Weiner, 1974) que la atribución de los resultados a la suerte tiene repercusiones sobre la motivación de logro. Se sabe que en los adultos la estructura de la tarea, el patrón de resultados obtenidos y lo inusual de un éxito o un fracaso constituyen la base para que el sujeto adulto atribuya los resultados a la suerte. No se ha estudiado directamente si el comportamiento de los niños en contextos relacionados con el logro es igual o diferente al que acabamos de señalar en los adultos. No obstante, los estudios de Piaget e Inhelder (1951) sobre la evolución de la idea de azar nos ofrecen una base sobre la que fundamentar nuestras expectativas en este punto. Piaget organiza sus conclusiones en torno al argumento de que para identificar un conjunto de fenómenos como hechos aleatorios, primero ha de identificarse un conjunto de fenómenos que no sean aleatorios, un fondo no aleatorio en relación con el cual el azar pueda surgir como figura. Sólo cuando los pro- 
cesos cognoscitivos están lo bastante desarrollados como para ordenar y organizar lo intrínsecamente cierto, legal y predictible por medio de operaciones racionales, las cosas que son intrínsecamente inciertas, ilegales e impredictibles pueden ser aprehendidas como tales. Durante el período preoperacional, antes de los 7-8 años, el niño no diferencia entre lo aleatorio y lo no aleatorio. A partir de esta edad, con el acceso a las operaciones concretas, el niño comienza a distinguir progresivamente entre lo posible y lo cierto: el niño se da cuenta de que hay un ámbito sobre el que puede saber y otro sobre el que sólo puede suponer. Sin embargo, no es hasta aproximadamente los 12 años, con el acceso a las operaciones formales -en especial a la capacidad para pensar en términos de combinaciones y proporcionescuando el niño comprende plenamente la noción de azar.

Como consecuencia de lo anterior cabe esperar que el niño no atribuya los resultados a la suerte antes de los 11-12 años o, en caso de hacerlo, cabe esperar que tal atribución no tenga las mismas implicaciones motivacionales que para los adultos. Aunque la tendencia a atribuir los resultados a la suerte en función de la naturaleza de la tarea, el patrón de resultados o lo inusual del éxito o el fracaso puede que comience a los 7-8 años, no es probable que sea general hasta los 11-12 años.

\section{Comprender no es 10 mismo que usar. ¿Qué atribuciones hacen espontáneamente los niños?}

Los estudios revisados en relación con la evolución de las atribuciones se han centrado en el grado de comprensión que los niños tienen de las mismas. Sin embargo, el que comprendan un concepto no significa que lo utilicen espontáneamente. De ahí que para determinar si cabe esperar que un tipo de atribución dado influya en la motivación de logro sea necesario estudiar el grado en que es utilizado espontáneamente por los niños. Frieze y Snyder (1980) intentaron responder a la pregunta siguiente: ¿qué atribuciones causales utilizan los niños para explicar los éxitos y los fracasos? Examinaron sujetos de 6,5 a 7,5 años, de 8,5 a 9,5 y de 10,5 a
11,5 años. Los resultados de su estudio pusieron de manifiesto que los sujetos más pequeños daban pocas respuestas que se pudieran codificar mediante el código utilizado en el experimento (The Coding Scheme of Perceived Causality. Elig y Frieze, 1975). Uno de los resultados más importantes fue que las atribuciones parecen depender estrechamente de la situación, esto es, aunque todas las actividades sobre las que se preguntó a los niños se realizaban en la escuela (exámenes, expresión artística, deportes, cazar ranas), las causas aducidas en cada caso para explicar el éxito o el fracaso no eran las mismas. En el caso concreto del logro académico las causas aducidas con más frecuencia para explicar el éxito son esfuerzo y habilidad, y para explicar el fracaso, ausencia de esfuerzo. La causa "suerte» apenas se utiliza de modo espontáneo. Por otra parte, el fracaso se atribuye más a causas inestables que el éxito en los mayores, pero no en los pequeños. Parece haber un incremento evolutivo en el uso de causas inestables, en especial para explicar el fracaso.

Los resultados anteriores tienen algunas implicaciones importantes que hay que considerar. En primer lugar, dado que los niños atribuyen los resultados espontáneamente a causas como el grado de esfuerzo, de habilidad o de dificultad de la tarea, cabe pensar que dichas atribuciones puedan mediar en la motivación de logro. Tiene sentido, por consiguiente, estudiar la evolución de dichas atribuciones cara a determinar su relación con la motivación de logro. Por lo que se refiere a la "suerte", el hecho de aprobar o suspender un examen no ofrece por sí solo base para pensar que el resultado pueda deberse al tipo de suerte. Es lógico, por ello, que esta atribución no apareciese en el estudio de Frieze y Snyder. En consecuencia, a partir de dichos resultados no se puede afirmar que los niños no utilicen dicha atribución. Es un punto que hay que investigar.

En segundo lugar, el hecho de que las atribuciones dependan de la situación implica la necesidad de verificar empíricamente bajo qué condiciones es posible que se den atribuciones análogas antes de utilizar escalas para medir las tendencias atributivas de los sujetos y de hacer generalizaciones a partir de los resultados. 


\section{Conclusión}

En la tabla I resumimos el estado de los conocimientos relativos a la adquisición por parte del niño de los distintos componentes que participan en la dinámica de la motivación de logro, resumen basado en los estudios que acabamos de revisar. Tenemos que señalar que los límites de edad que se presentan en dicha tabla lo son a título de orientación, ya que existe una gran variabilidad individual por lo que se refiere a la edad en la que cada niño logra las distintas adquisiciones. No obstante, lo que sí parece ser común a todos los niños es la secuencia de las mismas.

TABLA I

Adquisición y evolución de los componentes en base a los cuales se estructura el dinamismo de la motivación de logro

\section{HASTA LOS 6,5-7 ANOS}

1. Incentivo:

- A partir de los 4 años los niños pueden distinguir el éxito del fracaso y percibir que ellos son la causa de los mismos (McClelland, 1958; Heckhausen, 1967, y Veroff, 1969).

\section{Dificultad de la tarea:}

- No son capaces de percibir distintos niveles de dificultad (Nicholls, 1978 b), excepto si los indicios están muy claros (McClelland, 1958) a partir de la estructura de la tarea y del patrón de resultados propios.

\section{Esfuerzo:}

- Carecemos de información sobre si los niños son capaces de inferir el grado de esfuerzo a partir de los indicios de esfuerzo (intensidad de dedicación a la tarea y persistencia en la misma).

- No distinguen el esfuerzo del resultado ni de la habilidad (Nicholls, 1978 b; Kun, Parsons y Ruble, 1974).

\section{Habilidad:}

- No son capaces de inferir la habilidad a partir de la secuencia anterior de resultados, al parecer por dificultades de integración de la información serial (Shaklee, 1976).

- No distinguen la habilidad del esfuerzo (Nicholls, 1978 b; Kun, Parsons y Ruble, 1974).

5. Suerte:

- Los niños de este grupo son incapaces de percibir lo aleatorio de los resultados y, en consecuencia, la noción de suerte (Inhelder y Piaget, 1951).

\section{Esquemas causales:}

- Son capaces de utilizar el «esquema gradual» (Kun, Parsons y Ruble, 1974).

- No son capaces de utilizar el esquema de «compensación», lo que implica una comprensión inadecuada de las relaciones entre esfuerzo y habilidad.

- En lugar del esquema de compensación utilizan el «esquema halo» (Kun, 1977).

\section{Expectativas:}

- No utilizan la información procedente de la historia de resultados anteriores para formar expectativas (Heckhausen, 1967; Parsons y Ruble, 1972; Shaklee, 1976; Parsons y Ruble. 1977; Nicholls, 1978 b).

- No obstante, parece que son capaces de esperar el éxito (Heckhausen y Roelofsen, 1962). 


\section{A PARTIR DE LOS 7-8 ANOS}

\section{Incentivo:}

- Se intensifica la reacción afectiva ante el fracaso, reacción que antes era mínima (Ruble, Parsons y Ross, 1976).

- A partir de los 8 años el 70 por 100 de los sujetos son capaces de percibir distintos niveles de incentivo ligados a distintos niveles de dificultad. A los 7, sólo el 50 por 100 (Nicholls, 1978 b).

\section{Dificultad de la tarea:}

- El 70 por 100 de los sujetos son capaces de percibir la existencia de distintos niveles de dificultad a partir del patrón de resultados desde los 7 años (Nicholls, 1978 b).

- Los niños son capaces de utilizar la información relativa a los resultados obtenidos por otros para inferir el nivel de dificultad (Veroff, 1969; Ruble, Parsons y Ross, 1976; Shaklee, 1976).

\section{Esfuerzo:}

- Los niños son capaces de distinguir esfuerzo y resultado como causa y efecto (Nicholls, 1978 b).

- Los niños son capaces de inferir el grado de esfuerzo realizado a partir de los indicios de esfuerzo (Nicholls, 1978 a).

- El 91 por 100 de los niños son capaces de inferir el esfuerzo realizado a partir de los indicios sobre el grado de habilidad (Karabenick y Heller, 1976).

4. Habilidad:

- Hay resultados contradictorios. Para Karabenick y Heller (1976), el 63 por 100 de los sujetos son capaces de inferir la habilidad a partir de los indicios de esfuerzo. Para Nicholls (1978 b), los niños siguen sin distinguir habilidad y esfuerzo, no siendo capaces de inferir aquella causa cuando el esfuerzo no es suficiente para explicar el resultado.

\section{Suerte:}

- Comienza a utilizarse la noción de suerte (Inhelder y Piaget, 1951).

- Si los resultados son inconsistentes con la falta de esfuerzo, se atribuyen a la suerte (Nicholls, 1978 a).

\section{Esquemas causales:}

- A partir de los 8 años los niños son capaces de utilizar el esquema de «compensación inversa" e inferir el esfuerzo a partir de la habilidad (Kun, 1977).

\section{Expectativas:}

- Comienzan a ser capaces de formarse expectativas realistas a partir del patrón de resultados (Heckhausen, 1967; Parsons y Ruble, 1972; Nicholls, 1975; Shaklee, 1976; Nicholls, 1978).

\section{A PARTIR DE LOS 10-11 AÑOS}

\section{Habilidad:}

- Según Nicholls (1978 b) es a esta edad cuando comienza a inferirse la habilidad a partir del esfuerzo. En la misma línea están los resultados de Kun (1977).

\section{Suerte:}

- Perciben claramente lo aleatorio de los resultados, siendo capaces de inferir su dependencia del azar y la suerte (Inhelder y Piaget, 1951).

6. Esquemas causales:

- Son capaces de utilizar correctamente el esquema de compensación inversa (Kun, 1977).

\section{Expectativas:}

- Varían monotónicamente en función del éxito, pero no del fracaso (Parsons y Ruble, 1977). 
Alonso TÁıı, J.: "Atribución de la causalidad y motivación de logro». Tesis doctoral no publicada. Universidad Autónoma de Madrid. Madrid, 1983 (a).

A LONSO TAPıA, J.: «Atribución de la causalidad y motivación de logro. I: Estudio evolutivo de la utilización de información en la realización de juicios de atribución». Estudios de Psicología, 1983, 16, 13-27 (b).

AtKINSON, J. W.: «An introduction to motivation». Nueva York. Van Nostrand, 1964.

BERZONSKI, M.: «The role of familiarity in children's explanations of phisical causality". Child Development, $1971,42,705-715$.

CALLARD, E.: «Achievement motive in the four-year old and its relationship to achievement expectancies of mothers». Tesis doctoral no publicada. Universidad de Michigan, 1964.

Feldman, S., y BUSH, D.: Cognitive development and social development. Hillsdale, N. J.: Erlbaum, 1978.

FRIEZE, I. H., y SNYDER, H. N.: «Children's beliefs about the causes of success and failure in school settings". J. of Educational Psychology, 1980, 72, 2, 186-196.

FYANS, J. R.: Achievement motivation: recent trends in theory and research. Plenum Press, Nueva York, 1980.

Gelman, R.: “Cognitive Development». Annual Review of Psychology, 1978, 29, 297-332.

HeCKHAUSEN, H.: The anatomy of achievement motivation. Academic Press. Nueva York, 1967.

HECKHAUSEN, H., y RoElOFSEN, I.: «Anfänge und Entwicklung der Leistungsmotivation: (I) im Wetteifer des Kleinkindes». Psicbolo. Fosch., 1962, 26, 313-397.

HECKHAUSEN, H., y WAGNER, I.: «Anfänge und Entwicklung der Leistungsmotivation: (II) in der Zielsetzung des Kleinskindes. Zur Genese des Anspruchniveans". Psychol. Forch., 1965, 28, 179-245.

HeCkHAusen, H., y WASNA, M.: «Erfolg und Misserfolg in Leistungs wetteifer des imbezillen Kindes». Psychol. Forch., 1965, 28, 391-421.

HEIDER, F.: The psychology of interpersonal relations. Wiley. Nueva York, 1958.

KARABENick, J. D., y Heller, K. A.: «A developmental study of effort and ability attributions». Developmental Psychology, 1976, 22, 51-52.

KAssin, S. M.: «From laychild to layman: Developmental causal attribution». En: S. S. Brehm, S. M. Kassin, y F. X. Gibbons: Developmental social psycbology. Univ. Press. Oxford, 1981.

KELLE, H.: "Causal schemata and the atribution process». En E. E. Jones, D. E. Kanouse, H. Kelley, R. E. Nisbett, S. Valins, y B. Weiner: Attribution: perceiving the causes of bebavior. General Learning Press. Morristown, 1972.

KOHLBERG, L.: "Stage and sequence: the cognitive developmental approach to socialization». En D. Goslin: Handbook of socialization. Rand MacNelly. Chicago, 1971.

KUN, A.: "Development of the magnitude-covariation and compensation schemata in ability and effort attributions of performance». Cbild Development, 1977, 48, 862-873.

Kun, A.; Parsons, J. E., y Ruble, D. N.: «Development of integration processess using ability and effort

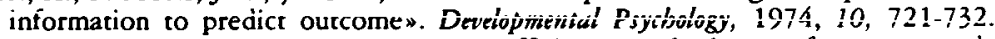

KUN, A., y WEINER, B.: «Necessary versus sufficient causal schemata for success and failure".J. of Research in Personality, 1973, 7, 197-202.

MCClelland, D. C.: "Risk taking in children with high and low need for achievement". En J. W. Atkinson: Motives in fantasy, action and society. Van Nostrand, Princeton, 1958.

MENDELSON, R., y SHULTL, T. R.: «Covariation and temporal contiguity as principles of causal inference in young children».J. of Experimental Child Psychology, 1976, 22, 408-412.

Nicholis, J. G.: «Causal attributions and other achievement related cognitions: effects of task outcome, attainment value and sex». J. of Personality and social Psycbology, 1975, 31, 379-389.

Nichous, J. G.: «Development of causal attributions and evaluative responses to success and failure in mahori and pakehna children». Developmental Psychology, 1978 (a), 14, 6, 687-688.

Nicholls, J. G.: "The development of the concepts of effort and ability, perception of accademic attainment and the understanding thar difficult tasks attainment and the understandings tath difficult task require more abilitym. Child Development, 1978, 49, 800-814.

Parsons, J. E., y Ruble, D. N.: «Attributional processess related to the development of achievement related affect and expectancy". Artículo presentado al encuentro anual de la APA, Hawaii, 1972.

Parsons, J. E., y Ruble, D. N.: “The development of achievement related expentacies». Child Development, 1977, 48, 1075-1079.

Parsons, J. E., Ruble, D. N.; Hodges, K. L., y Smali, A.: "Cognitive developmental factore in emerging sex differences in achievement related expectanciesn. Articulo no publicado. Smith College, 1975.

Pinget, J., e Inelder, B.: Le genese de l'idée de hasard chez l'enfant. PUF, París, 1951.

ROTTER, N. W.: «Social Learning and clinical psychology». Prentice Hall. Englewood Cliffs, 1954.

Ruble, D. N.; PArsons, J. E., y Ross, J.: «Self evaluative responses of children in an achievement setting". Child Development, 1976, 47, 990-997.

SHAKLEE, H.: «Development in inferences of ability and task difficulty». Child Development, 1976, 47, $1051-1057$.

Shult/, T.R., y MENDELSON, R.: "The use of covariation as a principle of causal analysis". Child Development, $1975,46,394-399$.

VEROFF, J.: "Social comparison and the development of achievement motivation". En Ch. P. Smith: Achievement-related motives in children. Rusell Sage Fundation. Nueva York, 1969.

WEINER, B.: "Achievement motivation as conceptualised. by and attribution theorist". En B. Weiner: Acbietement motitation and attribution theory. General Learning Press. Morristown, 1974.

WEINER, B.: Human motitation. Holt, Rinehart y Winston. Nueva York, 1980.

WONG, T. P., y WEINER, B.: "When people ask 'why' questions and the heuristics of attributional search". $J$. of Personality and Social Psychology, 1981, 40, 4, 650-663.

ZuniCh, M.: "Children's reaction to failure".J. Genet. Psychol., 1964, 104, 19-24. 


\section{Resumen}

En el presente trabajo se revisan los principales estudios realizados sobre el desarrollo de las condiciones que determinan la motivación de logro: percepción de los resultados como éxitos o fracasos; capacidad para distinguir distintos niveles de incentivo; comprensión de los conceptos de babilidad, esfuerzo, dificultad de la tarea y suerte, asi como de las relaciones compensatorias existentes entre ellos, y capacidad para formar expectativas realistas. También se revisan los estudios sobre las atribuciones que los niños realizan espontáneamente. Se indican tanto las implicaciones psicológicas y pedagógicas de los cambios evolutivos observados como los aspectos evolutivos que necesitan ser investigados.

\section{Summary}

In this article, the main studies on the development of conditions underlying acbievement motivation are revised, conditions sucb as: the perception of outcomes as successes orfailures, the capacity to distinguist among different incentive levels, the comprebension of the main attributional concepts -ability, effort, task difficulty and luck- and tbeir compensatory relations, and the capacity to form realistic expectancies. Studies about children's spontaneous attributions are also revised. Psycbological and educational implications from the developmental changes observed are suggested, and some issues to be investigated are indicated.

\section{Résumé}

Dans cet article on révise les études les plus importants sur le développement des conditions determinantes de la motivation pour la réussite: lo perception des résultats comme "riussites» ou "écbecs», la capacité pour distinguer des differentes niveaux daiguillon, la comprébension des concepts atributifs plus importants - babileté, effort, diffículté de la tâcbe et basard de mêtme que les relations de compensation entre ces concepts, et la capacité pour former des expectatives avec du réalisme. Des études sur les attributions spontanées des enfants sont aussi révisés. On montre tant les implications psychologiques et pédagogiques des cbangements evolutifs, aussi bien que les aspects du developpement sur lesquels on a besoin dapprofondir. 